\title{
\begin{tabular}{|l|l|l|l|l|}
\hline $\mathrm{M}$ & $\mathrm{R}$ & $\mathrm{S}$ & Internet Journal of & Nitride Semiconductor Research \\
\hline
\end{tabular}
}

Volume 1, Article 20

\section{Recent Results in the Crystal Growth of GaN at High $\mathbf{N}_{2}$ Pressure}

\author{
I. Grzegory, M. Bockowski, B. Lucznik, S. Krukowski, M. Wroblewski, S. Porowski \\ High Pressure Research Center
}

This article was received on May 31, 1996 and accepted on October 23, 1996.

\begin{abstract}
We present recent results on bulk GaN crystallization. The best quality GaN crystals grown from the solution at high $\mathrm{N}_{2}$ pressure without an intentional seeding are single crystalline platelets of stable morphology reaching dimensions up to $10 \mathrm{~mm}$. The fastest growth direction for such crystals is $\left[\begin{array}{llll}1 & 0 & \overline{1} & 0\end{array}\right]$, perpendicular to the GaN c-axis. The maximum stable growth rate perpendicular to crystal c-axis is determined from the experiment and used for an estimate of the effective supersaturation for the $\{10 \overline{1} \quad 0\}$ face assuming two dimensional layer growth. The heat of GaN disssolution, determined from experimental solubility data, is used for the estimation of the edge energy of 2-D nuclei on the growing $\{10 \overline{1} \quad 0\}$ face. Bulk crystal growth seeded by a single hexagonal needle with well developed $\{10 \overline{1} \quad 0\}$ faces is also reported. The crystallization mechanisms and morphological stability in seeded growth of $\mathrm{GaN}$ are discussed on the basis of experimental results. The physical properties of the GaN crystals and homoepitaxial layers grown on them are briefly reviewed.
\end{abstract}

\section{Introduction}

GaN is a strongly bonded compound well suited for applications such as high power, high temperature electronics. The bonding energy of GaN is $8.9 \mathrm{eV} /$ atom whereas for $\mathrm{GaAs}$ it is only $6.5 \mathrm{eV} /$ atom [1]. Consequently, the free energy $G(T)$ of the crystal is very low in relation to the reference state of free $N$ and $G a$ atoms. On the other hand, the $\mathrm{N}_{2}$ molecule is also strongly bonded (4.9eV/atom). Therefore, the free energy of the constituent $\mathrm{Ga}$ and $\mathrm{N}_{2}$ becomes quite close and surpasses that of $\mathrm{GaN}$ as the temperature is raised. Figure 1 shows the free energy of $\mathrm{GaN}$ ( 1 mole) versus its constituents $\left(\mathrm{Ga}+1 / 2 \mathrm{~N}_{2}\right)$ as a function of temperature.

The $\mathrm{G}(\mathrm{T})$ of the constituents decreases faster than that of the GaN crystal and at higher temperatures GaN become thermodynamically unstable. The crossing of $G(T)$ curves determines the equilibrium temperature where $\mathrm{GaN}$ coexists with its constituents at a given $\mathrm{N}_{2}$ pressure. The application of pressure allows us to increase the free energy of the constituents much more than that of the crystal shifting the equilibrium point to higher temperature and extending the stability range of GaN. The equilibrium $\mathrm{p}_{\mathrm{N} 2}-\mathrm{T}$ conditions for GaN determined experimentally by Karpinski et al. [2] [3] are shown in Figure 2.

Most of crystallization processes discussed in this paper have been carried-out in large volume gas pressure reactors at an $\mathrm{N}_{2}$ overpressure of up to $15 \mathrm{kbar}$, which corresponds to a GaN stability limit at $1850 \mathrm{~K}$. The maximum temperature of $1850 \mathrm{~K}$ is quite far from the melting temperature of GaN. According to the theoretical estimation of Van Vechten [4] the melting point of $\mathrm{GaN}$ is $\sim 3000 \mathrm{~K}$. The $\mathrm{N}_{2}$ equilibrium pressure at the melting point is therefore much higher than we can obtain in our experimental system. The extension of the GaN stability range to $1850 \mathrm{~K}$ allows the growth of GaN crystals from a liquid Ga solution. In Figure 3 we show the $\mathrm{N}$ solubility data resulting from the annealing of $\mathrm{Ga}$ at the three phase equilibrium conditions of Figure 2.

Since the $\mathrm{N}$ concentrations are not high, the growth experiments have to be long to obtain high quality bulk GaN 
crystals with dimensions appropriate for measurements and applications. Therefore, stability over a long time $(>100 \mathrm{~h})$ is an additional requirement for the growth system. The solid line in Figure 3 is the liquidus line for Ga-GaN system calculated using an ideal solution approximation and Van Vechten's melting temperature of 2790K. For an ideal solution, the solubility can be expressed as:

$$
n=n_{0} \exp \left(\frac{\Delta H_{\mathrm{ool}}}{k T}\right)
$$

where $\mathrm{H}_{\mathrm{sol}}$ is the heat of dissolution. For $\mathrm{GaN}, \mathrm{H}_{\mathrm{sol}}=44.7 \mathrm{kcal} / \mathrm{mole}=0.49 \mathrm{eV} / \mathrm{bond}$ and expresses the bonding energy in the crystal in relation to its mother phase: the solution.

\section{Crystal Growth}

\subsection{Experimental}

The growth system is designed for the crystallization of $\mathrm{GaN}$ from solution under high $\mathrm{N}_{2}$ overpressure at high temperature in relatively large volumes. Since the material to be grown is a semiconductor for optoelectronic applications, the system has to fullfil all the requirements concerning purity and control of the growth conditions. Moreover, crystallization from a dilute solution requires lengthy processes of $\sim 100$ hours. Therefore, the high pressure - high temperature crystallization reactor (chamber and multizone furnace) has to be supplied with additional systems necessary for:

1. in situ annealing in vacuum

2. electronic stabilization and programming of pressure

3. electronic stabilization and programming of temperature

4. pressure, temperature, voltage and current sensitive alarms

5. cooling of the pressure chamber

In Figure 4 we have shown schematically such an experimental apparatus. Many functions mentioned above are realized by the use of standard commercially available devices ( e.g. vacuum pumps, temperature programmers, mass flow detectors...). The pressure in the chamber is stabilized with a precision better than 20 bar. The temperature is measured by a set of thermocouples arranged along the furnace and coupled with the standard input power control electronic system. This allows the stabilization of temperature better than 0.5 deg., and the ability to program changes in the furnace temperature distribution during the experiment.

Supersaturation in the growth solution has been created by the application of temperature gradients along the axis of the crucible. Both horizontal and vertical arrangements of the crucibles have been used. The typical duration of the experiments was $100-150$ hours for crystals of 6-10 mm in size.

\subsection{Crystallization without intentional seeding}

Gallium, when heated under high $\mathrm{N}_{2}$ pressure, does not wet the crucible walls (usually graphite or BN). Rather, it is covered with a thin $\mathrm{GaN}$ layer consisting of $\mathrm{GaN}$ single crystals. This is the first step of $\mathrm{GaN}$ crystallization at high $\mathrm{N}_{2}$ pressure. The size of the crystals covering the Ga depends on the sequence and rates of compression and heating of the system. A typical structure of the surface layer is shown in Figure 5.

The formation of the surface layer inhibits further synthesis of the nitride which allows longtime crystallization processes and the growth of relatively large GaN crystals. Since the solution is in a temperature gradient, the GaN layer at the hot end of the crucible dissolves in gallium and is transported, by convection and diffusion, to the cooler zone of the crucible where the solution become supersaturated and crystallization of GaN takes place. The crystals of the surface layer are the natural source of growth centers for further crystallization. Some of them start to grow faster hindering the growth of the neighboring ones. This selection leads to the formation of relatively large, well shaped GaN crystals on the internal surface of the surface layer.

The GaN crystals grown by this method are wurzite, mainly in the form of hexagonal platelets or hexagonal needles, depending on the growth conditions. A general tendency is that high supersaturation favors growth in the 
c-direction which produces the needle-like crystals. Supersaturation of the growth solution is determined mainly by the growth temperature, supercooling, mass transport mechanisms in $\mathrm{Ga}$, and also the local surroundings for a particular crystal.

Crystals in the form of hexagonal platelets grow slowly, with a rate $<0.1 \mathrm{~mm} / \mathrm{h}$ into the $\{10 \overline{1} \quad 0\}$ directions (perpendicular to the c-axis). They are usually single crystals having perfect morphology suggesting stable layer-by layer growth. They are transparent, colorless, with flat faces. In some cases, three dimensional, hexagonal growth which occurs during the post-growth system cooling is observed on the crystal surfaces. Some GaN platelets grown without intentional seeding are shown in Figure 6.

For crystallization of large GaN crystals by this method, it is crucial to control the supersaturation to avoid acceleration of the growth near the edges and corners of the growing crystal. This can occur if some of them reach dimensions significantly larger than the others. Then the edge nucleation on the hexagonal faces of the $\mathrm{GaN}$ platelets is often observed. This is the first step to unstable growth on these faces. The result of such a growth is shown in Figure 7a. In extreme cases of very high supersaturation or when the supersaturation is abruptly increased (i.e. by increasing the temperature gradient), the growth into c-direction, nucleated at the edges of the platelet, become very fast and leads to the formation of well developed $\{10 \overline{1} \quad 0\}$ faces. This results in a hollow needle-like crystal such as is shown in Figure $7 \mathrm{~b}$. Therefore, for growing of large GaN platelets of stable morphology, the supersaturation must be decreased during the crystallization process inorder to prevent the runaway phenomena just described.

Therefore for the stable growth of large GaN crystals it is necessary to have sufficiently low and uniform supersaturation across the growing crystal faces. One of the most important factors influencing the uniformity of $\mathrm{N}$-concentration in the growth zone is the volume of the solution in relation to the size of the growing crystal. In Figure 8 we show a crystal grown in a crucible with an internal diameter of $17 \mathrm{~mm}$ (as opposed to $14 \mathrm{~mm}$ previously). The increase in the crucible volume resulted in a significant increase of the size of crystals which could be grown with stable morphology.

We summarize the general tendencies of GaN crystal growth without an intentional seeding following from the experimental results:

1. The growth of GaN crystals from a diluted solution in liquid gallium is strongly anisotropic.

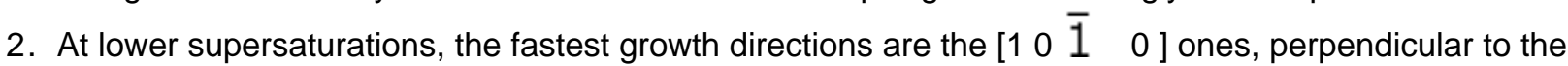
$\mathrm{c}$-axis of the crystal. The growth rate in the $\left[\begin{array}{llll}0 & 0 & 0 & 1\end{array}\right]$ directions, parallel to the c-axis is then extremely slow. This results in thin hexagonal platelets.

3. With increasing supersaturation, the rate of the growth parallel to the c-axis abruptly increases, resulting in needle like crystals with well developed $\{10 \overline{1} \quad 0\}$ faces.

We have estimated the supersaturation at which high quality GaN platelet grow at a rate of $0.1 \mathrm{~mm} / \mathrm{h}$. in the [1 $0 \overline{1}$ 0] direction (see Figure 9). For the estimation, we assumed two dimensional layer-by-layer growth on the $\{10 \overline{1} 0\}$ face of the platelet with a thickness of $0.1 \mathrm{~mm}$ and length of the leading edge of $1 \mathrm{~mm}$. We also assumed that the creation of a 2-D critical nucleus on the crystal face results in the growth of a new layer. That is, the lateral growth rate is fast enough to complete the plane before appearance of the next nucleus of the critical size. A heat of GaN dissolution in liquid $\mathrm{Ga}$ of $0.49 \mathrm{eV} / \mathrm{bond}$ (see Introduction) was used as a measure of bond energy in the crystal in relation to the liquid phase.

For a crystal growing at $1800 \mathrm{~K}$, we have obtained a supersaturation of the order of $50 \%$. For the growth of GaN on a seed crystal with well developed $\{10 \overline{1}$ 0\} faces, the supersaturation necessary for stable two dimensional growth can be significantly lower due to large surface area of the fast growing planes. The details of this estimation will be published elsewhere [5].

\subsection{Seeding}

Knowing that $\mathrm{GaN}$ in our experimental conditions prefers to grow in a stable way in directions perpendicular to the c-axis, we decided to use a needle like crystal to seed the growth on the $\{10 \overline{1} \quad 0\}$ faces. In Figure 10, we show the seed crystal which was introduced into the cooler zone of the vertical crucible. It had well developed $\{10 \overline{1} 0\}$ 
prismatic faces and also $\{1011\}$ pyramidal ones. A hexagonal surface did not appear due to the fast growth of the needle in c-direction.

Prior to the growth, it was necessary to heat the system up to $1200^{\circ} \mathrm{C}$ at low $\mathrm{N}_{2}$ pressure to achieve wetting of the seed with liquid $\mathrm{Ga}$. If the sysyem were be heated at high pressure, the polycrystalline surface layer would cover the Ga surface adjacent to the seed, and eliminate its usefulness for further crystallization.

The growth on the seed was performed under conditions where high quality platelets were obtained in previously described processes without intentional seeding. The resulting crystal, after 50 hours of growth, is shown in Figure 11.

The fast growth directions were the $\left[\begin{array}{lllll}1 & 0 & \overline{1} & 0\end{array}\right]$, as expected, and also the $\left[\begin{array}{llll}1 & 0 & \overline{1} & 1\end{array}\right]$ one perpendicular to the pyramidal face. Since the growth rate in the c-direction was very small, a hexagonal face developed in the resulting crystal.

As we know from previous considerations, at low supersaturations, the growth rate in c-direction is almost zero whereas at higher supersaturations we fall into the "difficult" region of the abrupt increase of the nucleation rate. Therefore, the growth on the hexagonal face is difficult to control and probably is very sensitive to fluctuations of temperature, convectional flows and uniformity of the nitrogen concentration field in the crystal surrounding. This can easily lead to three dimensional nucleation or to edge instabilities of the growth.

\section{Physical Properties of GaN crystals}

The quality of the GaN crystals was estimated by X-ray rocking curves [6]. The full width at half maximum (FWHM) of the X-ray rocking curves ((0004) CuK reflection) depends on the size of the crystal. The value is 20-30 arcsec for $1 \mathrm{~mm}$ crystals and 30-40 arcsec for 1-3 mm ones. For larger platelets the rocking curves split into a few 30-40 arcsec peaks showing the presence of low angle (1-3 arcmin) boundaries separating grains of $0.5-2 \mathrm{~mm}$ in size.

Misorientation between grains increases monotonically from end to end of the crystal [7]. This indicates a "bending" of the crystal during growth, followed by strain relaxation leading to the formation of the low angle boundaries. Indeed, the lattice constantsmeasured for the two basal planes of the crystals differ by $0.001 \approx$, suggesting different growth mechanisms and defect structures for the two polar directions of the GaN platelet. It was shown by Liliental-Weber et al. [8] by TEM examination and confirmed by AFM studies [9], that one of the polar surfaces of the GaN crystals (especially for the smaller ones) is often atomically flat (2-3 monolayer steps present) and the crystal below is practically free of extended defects. However a high concentration of point defects, mainly N-vacancies and Ga-antisites, were deduced from Convergent Beam Electron Diffraction patterns [8]. On the opposite side, regular pyramids of $100-200 \approx$ in size terminate the surface [8] [9]. Below are many extended defects such as stacking faults, dislocation loops and Ga micro-precipitates. Such pecularities result from the different growth modes of the two polar directions of the crystal in the Ga-rich conditions of the process.

For larger (>3 mm) GaN plateletes, the presence of three dimensional hexagonal features on the surfaces is typical. This results from the system cooling after the growth in the temperature gradient.

The GaN crystals are highly conductive, showing metallic behavior in the whole temperature range of 4.2-300K. Free electron concentration of $10^{19}-10^{20} \mathrm{~cm}^{-3}$ with mobilities of $30-90 \mathrm{~cm}^{2} / \mathrm{Vs}$ [10] are present regardless of temperature. No clear dependence of the electrical properties on the growth conditions has been observed. Both ab initio calculations of electronic structure of GaN native defects [11] [12] and experiments [13] confirm that the source of free electrons in this $\mathrm{GaN}$ is $\mathrm{N}$-vacancies.

The optical absorption spectra of pressure-grown GaN crystals and epitaxial GaN layers with a free electron concentration of $6 \times 10^{17} \mathrm{~cm}^{-3}$ have been measured by Teisseyre et al. [14]. A shift to high energy of the optical absorption edge for the bulk sample is observed and assigned to the Burstein-Moss effect [14]. Due to this shift, the bulk GaN substrate crystals are transparent to near edge luminescence of GaN.

The dominant luminescence features of GaN bulk crystals are near band edge emission at $~ 3.5 \mathrm{eV}$ and a broad yellow luminescence band centered around $2.3 \mathrm{eV}$.

\section{GaN homoepitaxy}

The first results of homoepitaxial growth of GaN MOCVD layers have been recently reported [15]. Pressure grown 
GaN crystals were used as substrates. The layers were grown in a horizontal atmospheric pressure Epigress system adapted to the growth of nitrides. TMG and $\mathrm{NH}_{3}$ were used as sources of $\mathrm{Ga}$ and $\mathrm{N}$, and $\mathrm{H}_{2}$ was the carrier gas. The substrates did not require any mechanical or chemical surface treatment before the growth. Also the deposition of a low temperature buffer layer was omitted. The growth temperature was $900-1050^{\circ} \mathrm{C}$. P-doping was realized using the $\mathrm{MCp} \mathrm{p}_{2} \mathrm{Mg}$ metalorganic and subsequent annealing of the layer at $700^{\circ} \mathrm{C}$ in the presence of $\mathrm{N}_{2}$ gas. Si was introduced to achieve n-doping of the layers.

Atomic Force Microscopy studies performed on homoepitaxially grown GaN layers indicate the presence of monatomic steps on the layer surfaces, often in the form of large diameter $(100 \mathrm{~m})$ spirals indicating two dimensional growth on screw dislocations.

The X-ray diffraction peaks from the homoepitaxial layer and from GaN substrate from [7] are shown in Figure 12. The lattice constant parallel to the interface is the same for both the substrate and homolayer, whereas the c constant of the epilayer is smaller by about $0.02 \%$. The increase of the c lattice constant of the bulk GaN crystals is related to the presence of the same point defects responsible for the high concentration of free electrons.

The high quality of the homoepitaxial layers is confirmed by the photoluminescence measurements [15]. The spectrum is dominated by two strong and narrow lines $\mathrm{XD}(3.4719 \mathrm{eV})$ and $\mathrm{XA}(3.4666 \mathrm{eV})$ related to the excitons bound to neutral impurity centers [15]. The FWHM of these lines is about $1.8 \mathrm{meV}$ and $1.0 \mathrm{meV}$ respectively. The higher energy XD line shows a double peak structure which fitted with Lorenzian curves can be described by energies: $\mathrm{E}_{1}=3.4711 \mathrm{eV}, \mathrm{E}_{2}=3.4720 \mathrm{eV}$. The photoluminescence and reflectivity of GaN homoepitaxial layers are analyzed in detail in [15]. Also the lifetime of the bound excitons has been measured [16] to be 650 and 700 ps for excitons bound to the neutral donor and acceptor, respectively. This is much higher than the corresponding values for $\mathrm{GaN} /$ sapphire (70 and $140 \mathrm{ps,} \mathrm{respectively).}$

The PL spectra for p-type Mg doped GaN homoepitaxial layers have been reported [17]. In addition to the XD and XA exciton lines (XA line much stronger than for undoped layers), a series of very narrow lines attributed to donor-acceptor pair related transitions have been observed between $3.4-3.5 \mathrm{eV}$. The FWHM of these lines were < $0.5 \mathrm{meV}$. The PL lines observed for MOCVD grown homoepitaxial layers are the narrowest so far reported for GaN.

Recently very high quality homoepitaxial layer have been obtained by MBE [18]. The nearedge photoluminescence spectrum of this layer is dominated by XD and XA peaks and 3 lines corresponding to free excitons. The PL lines for this layer are even sharper than for best MOCVD samples. The halfwidth of the XA line is smaller than $0.5 \mathrm{meV}$

\section{Conclusions}

High quality GaN crystals can be grown at nitrogen pressure up to $15 \mathrm{kbar}$. The main obstacle to growing larger substrate sized GaN crystals is instability of the growth. The instability is related to the non-uniform distribution of nitrogen in the solution due to the small volume of the crucible in relation to the size of growing GaN crystals.

It is shown that high quality homoepitaxial layers can be obtained on the "as grown", single crystalline, pressure grown GaN substrates. Further optimization of both substrate and homoepitaxial growth techniques should lead to the fabrication of efficient optoelectronic devices. Special effort has to be paid to increasing of the size of high quality GaN substrates.

\section{Acknowledgments}

The research reported in this work has been supported by the Polish Committee for ScientificResearch grant \# $7783495 \mathrm{C} / 2399$.

\section{References}

[1]W. A. Harrison, Electronic Structure and Properties of Solids, Freeman, San Francisco, (1980)

[2] J. Karpinski, J. Jun, S. Porowski, J. Cryst. Growth 66, 1 (1984).

[3] J. Karpinski, S. Porowski, J. Cryst. Growth 66, 11 (1984). 
[4] J. A. Van Vechten, Phys. Rev. B 7, 1479 (1973).

[5]l. Grzegory and S. Porowski, unpublished.

[6] M Leszczynski, I Grzegory, M Bockowski, J. Cryst. Growth 126, 601-604 (1993).

[7] M. Leszczynski, I. Grzegory, H. Teisseyre, T. Suski, M. Bockowski, J. Jun, S. Porowski, J. Crystal Growth, unpublished (1996).

[8] Z. Liliental-Weber, C. Kisielowski, S. Ruvimov, Y. Chen, J. Washburn, I. Grzegory, M. Bockowski, J. Jun, S. Porowski, J. Electron. Mater. 25, 1545 (1996).

[9] G. Nowak, S. Krukowski, I. Grzegory, S. Porowski, J.M. Baranowski, K. Pakula, J. Zak, MRS Internet J. Nitride Semicond. Res. 1, 5 (1996).

[10] P. Perlin , J. Camassel, W. Knap, T. Taliercio , J. C. Chervin, T. Suski, I. Grzegory, S. Porowski , Appl. Phys. Lett. 67, 2524-2526 (1995).

[11] P. Boguslawski, E. L. Briggs, J. Bernholc, Phys. Rev. B 51, 17255-17258 (1995).

[12] Jorg Neugebauer, Chris G. Van de Walle, Phys. Rev. B 50, 8067-8070 (1994).

[13] P. Perlin, T. Suski, H. Teisseyre, M. Leszczynski, I. Grzegory, J. Jun, S. Porowski, P.Boguslawski, J. Bernholc, J. C. Chervin, A. Polian, T. D. Moustakas, Phys. Rev. Lett. 75, 296-299 (1995).

[14] H. Teisseyre, P. Perlin, T. Suski, I. Grzegory, S. Porowski, J. Jun , A. Pietraszko, T. D. Moustakas, J. Appl. Phys. 76, 2429-2434 (1994).

[15] K Pakula, A Wysmolek, KP Korona, JM Baranowski, R Stepniewski, I Grzegory, M Bockowski, J Jun, S Krukowski, M Wroblewski, S Porowski, Sol. St. Comm. 97, 919-922 (1996).

[16] M. Godlewski, A. Wysmolek, K. Pakula, J. M. Baranowski, I. Grzegory, J. Jun, S. Porowski, J. P. Bergman, B. Monemarin Proc. of Intl. Symp. on Blue Lasers and Light Emitting Diodes, , (Chiba, Japan, March 5-7, , 1996) 356.

[17] A. Wysmolek, J. M. Baranowski, K. Pakula, K. P. Korona, I. Grzegory, M.Wroblewski, S. Porowskiin Proc. of Intl. Symp. on Blue Lasers and Light Emitting Diodes, , (Chiba, Japan, March 5-7, , 1996) 492.

[18] H. Teisseyre, G. Nowak, M. Leszczynski, I. Grzegory, M. Bockowski, S. Krukowski, S. Porowski, M. Mayer, A. Pelzmann, Markus Kamp, K. J. Ebeling, G. Karczewski, MRS Internet J. Nitride Semicond. Res. 1, 13 (1996). 


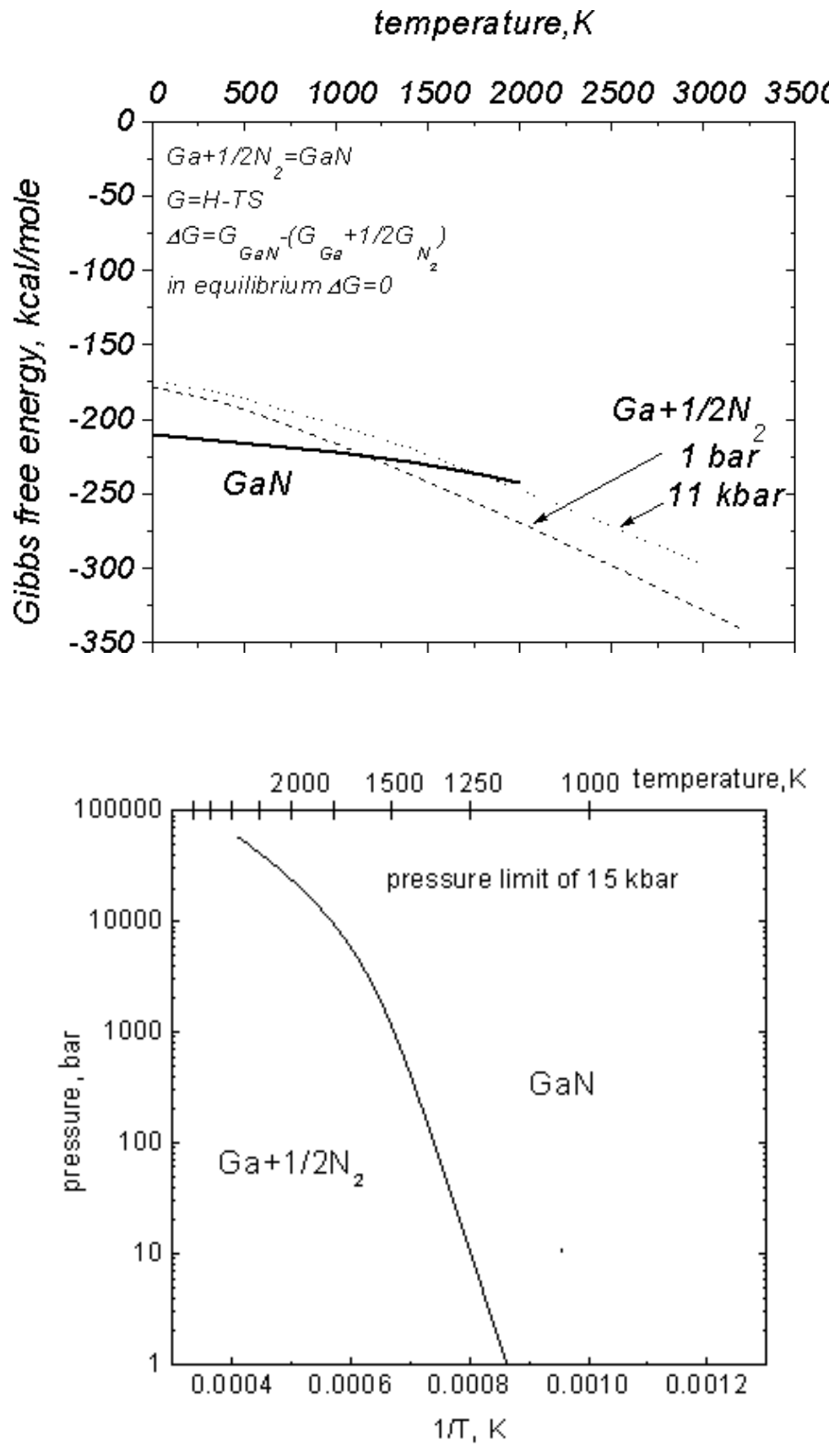

Figure 1. Gibbs free energy of GaN and its constituents

Figure 2. Equilibrium curve for GaN [2] [3] 


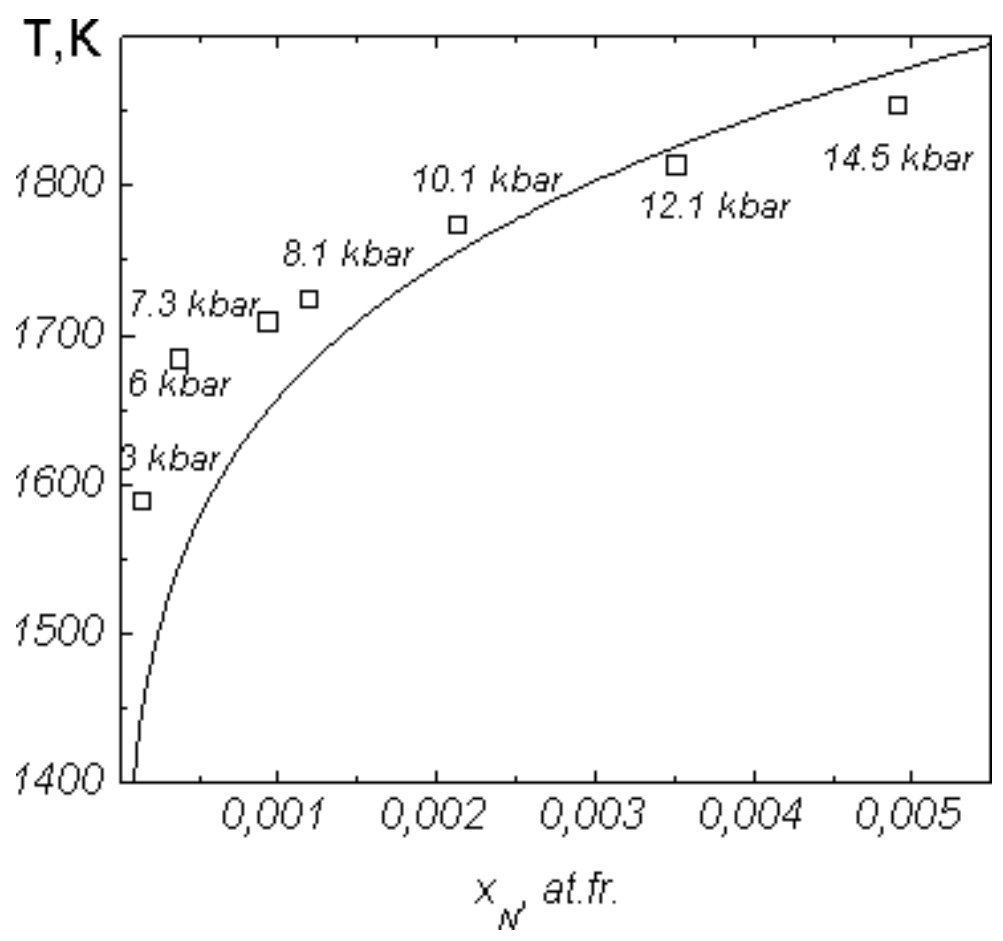

Figure 3. Liquidus line for the Ga-GaN system

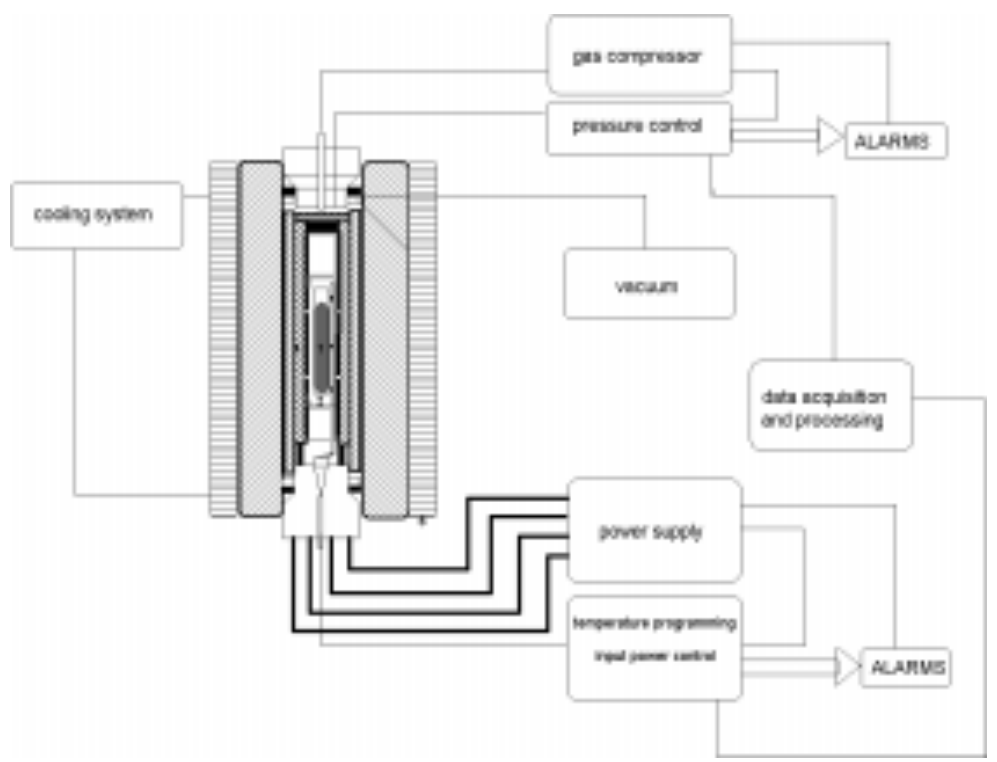

Figure 4. Experimental system for the crystal growth of $\mathrm{GaN}$ at $\mathrm{N}_{2}$ pressure up to $15 \mathrm{kbar}$. 


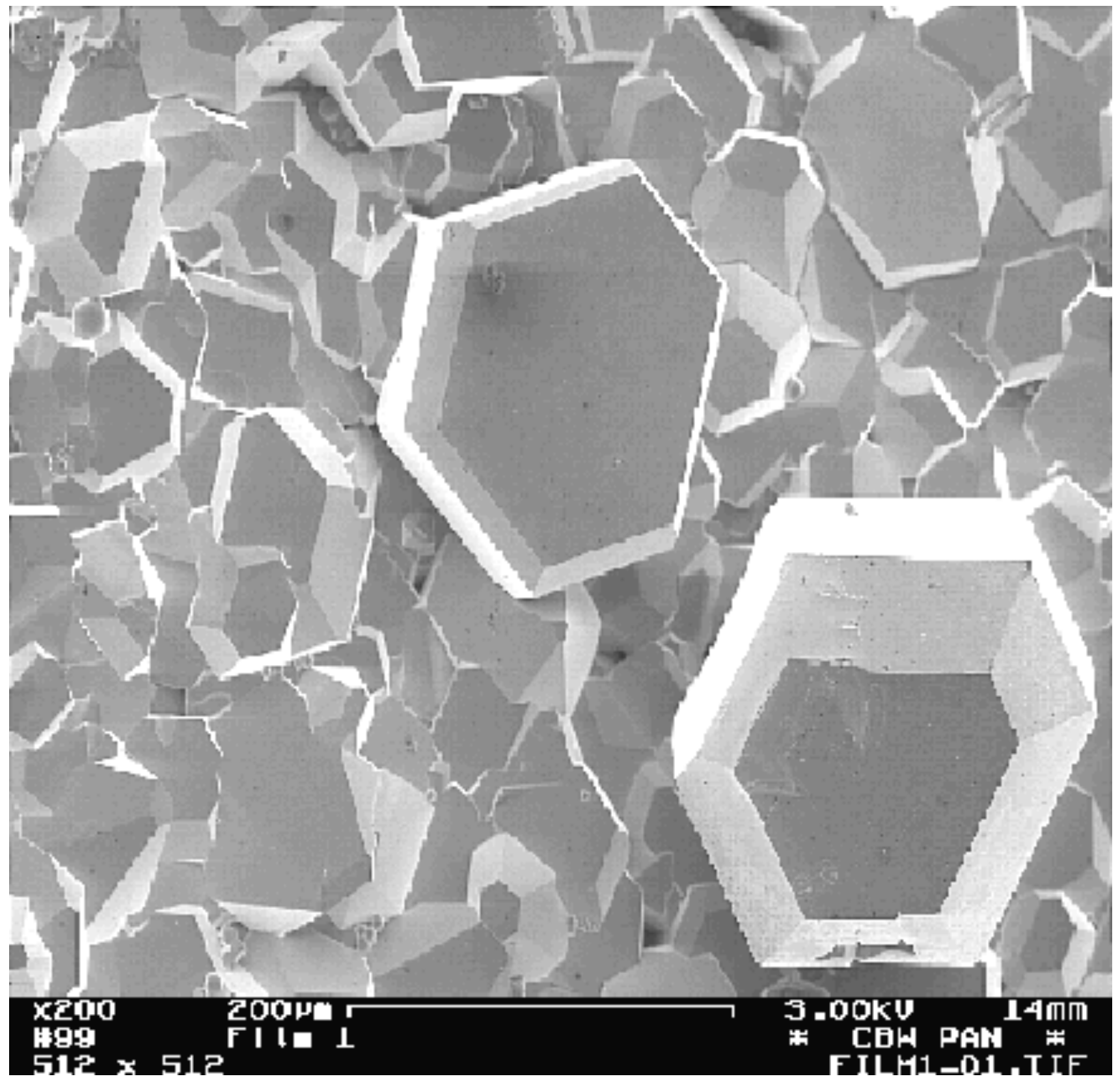

Figure 5. Crystallized GaN surface layer at high $\mathrm{N}_{2}$ pressure 


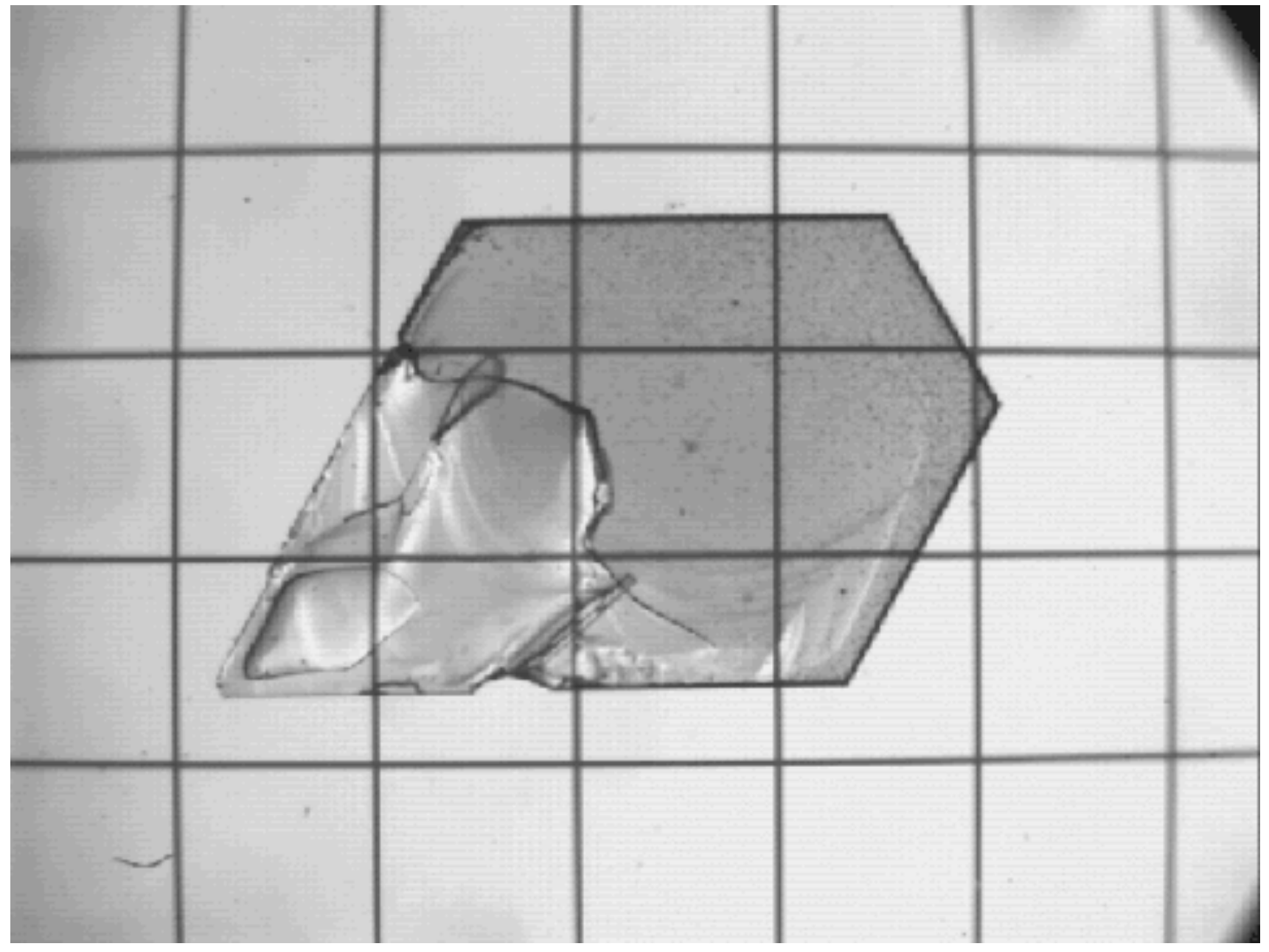

Figure 6a. GaN single crystals grown without an intentional seeding. The underlying grid is $1 \mathrm{~mm} \times 1 \mathrm{~mm}$. 


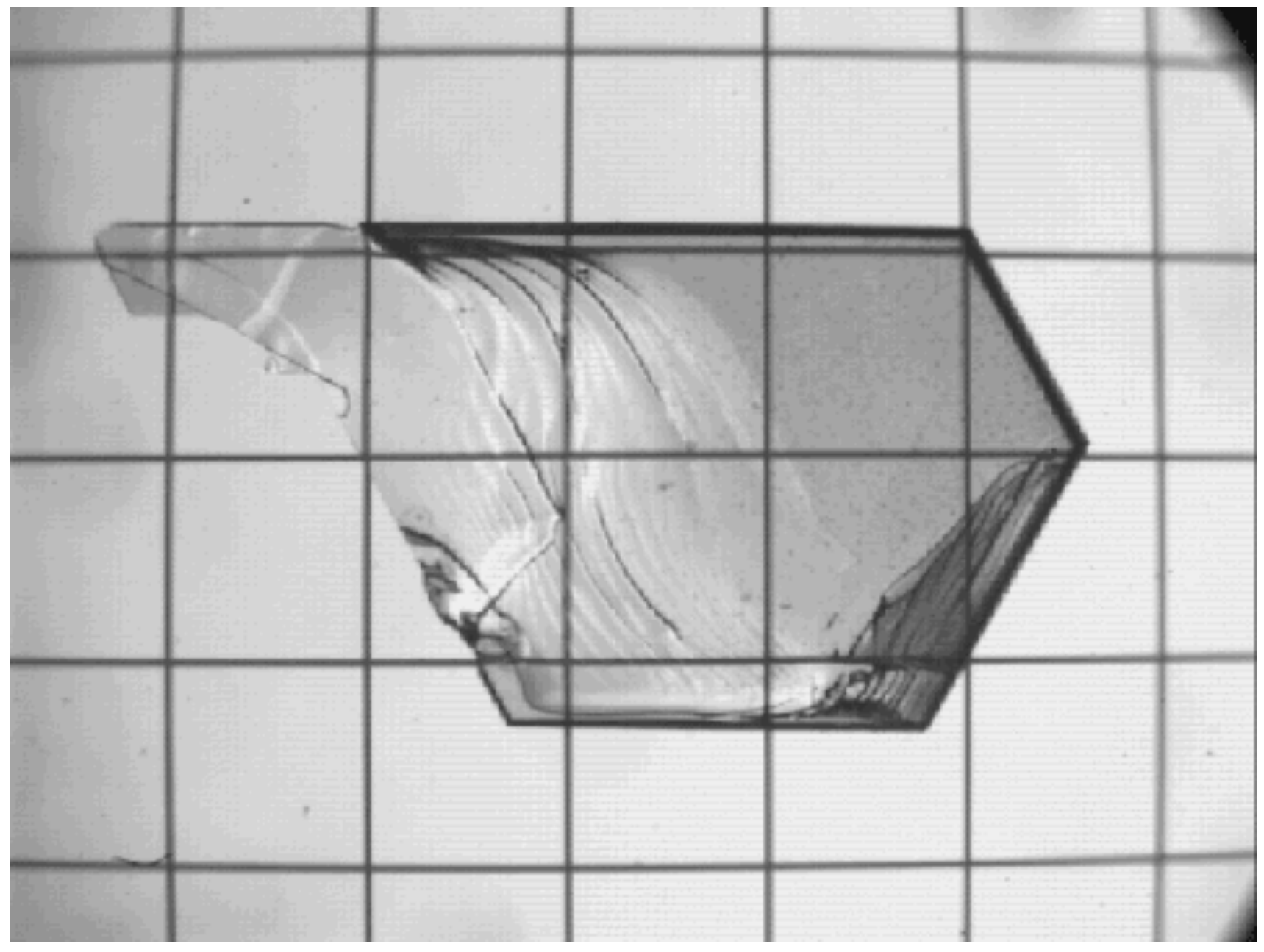

Figure 6b. 


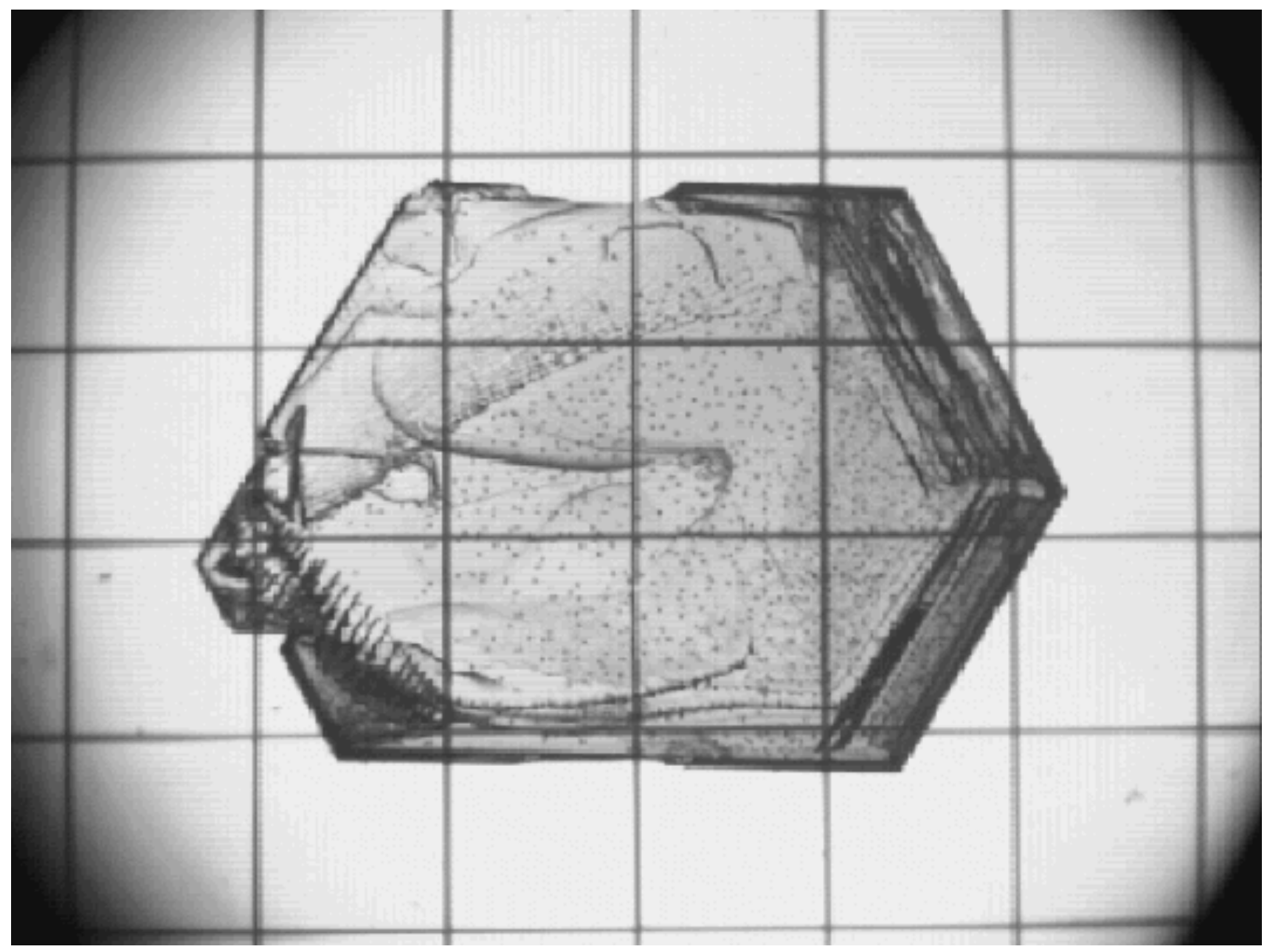

Figure 6c. 


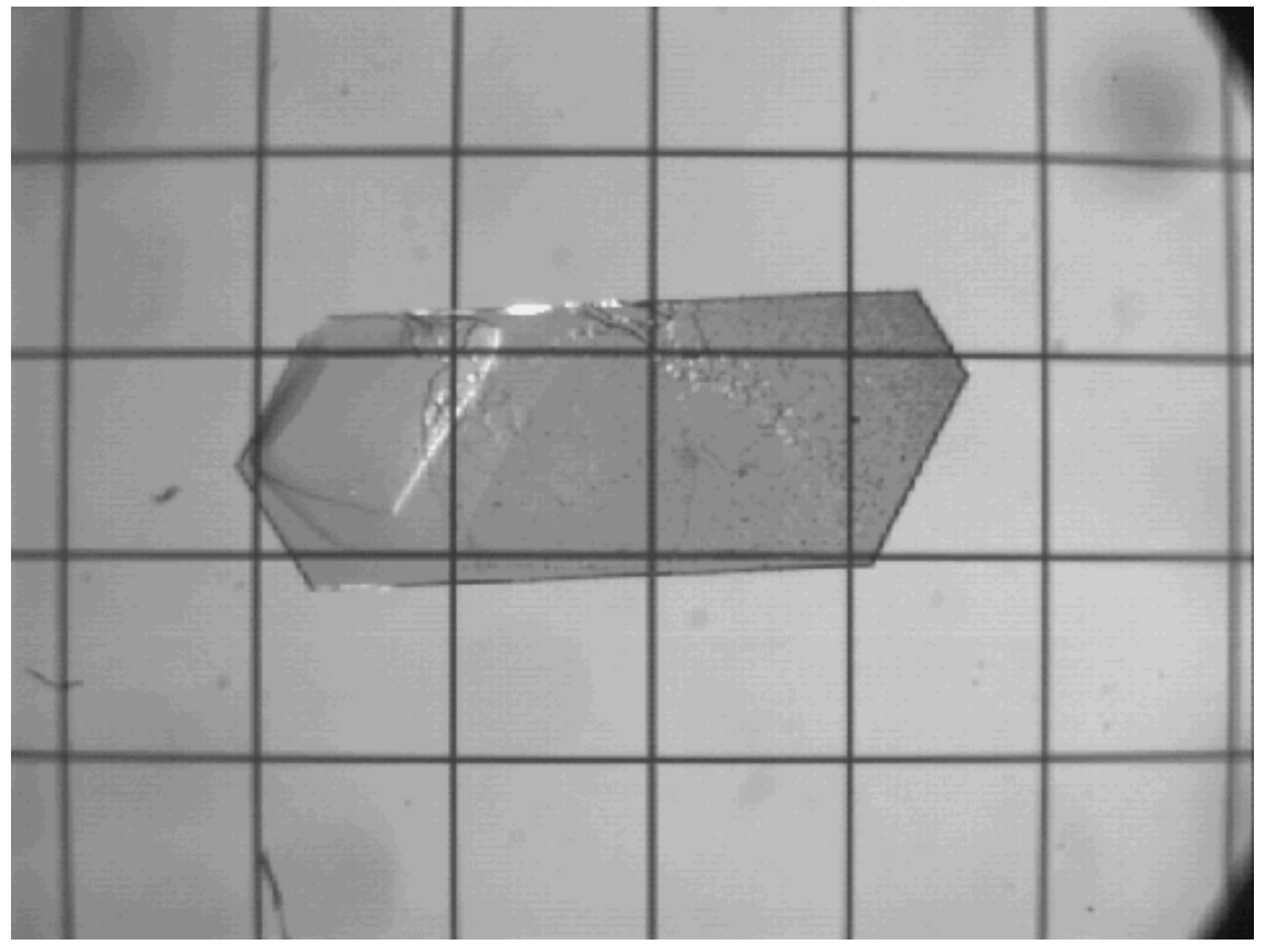

Figure 6d. 


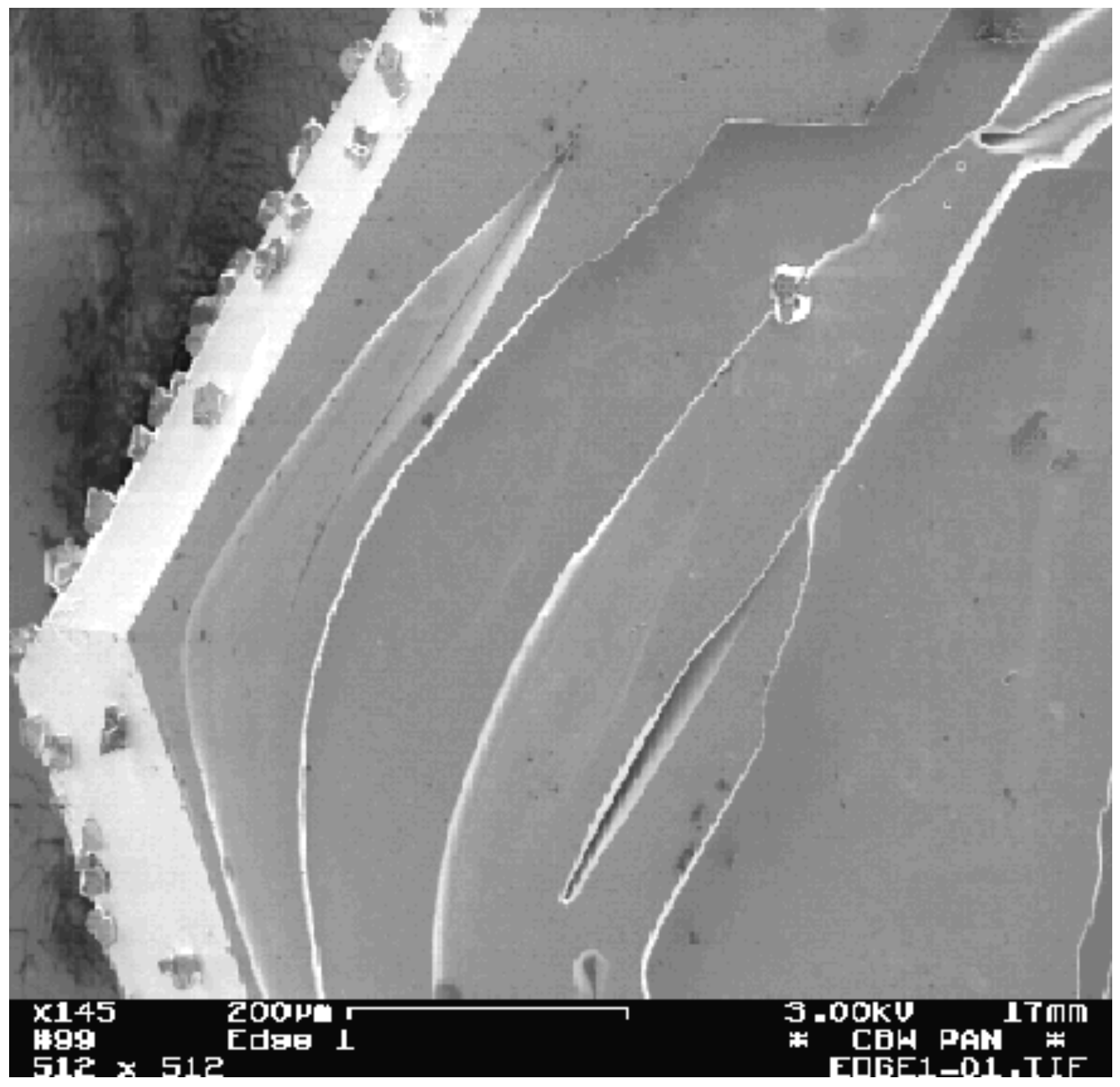

Figure 7a. Edge-nucleated growth on the $\{0001\}$ faces at high supersaturation. 


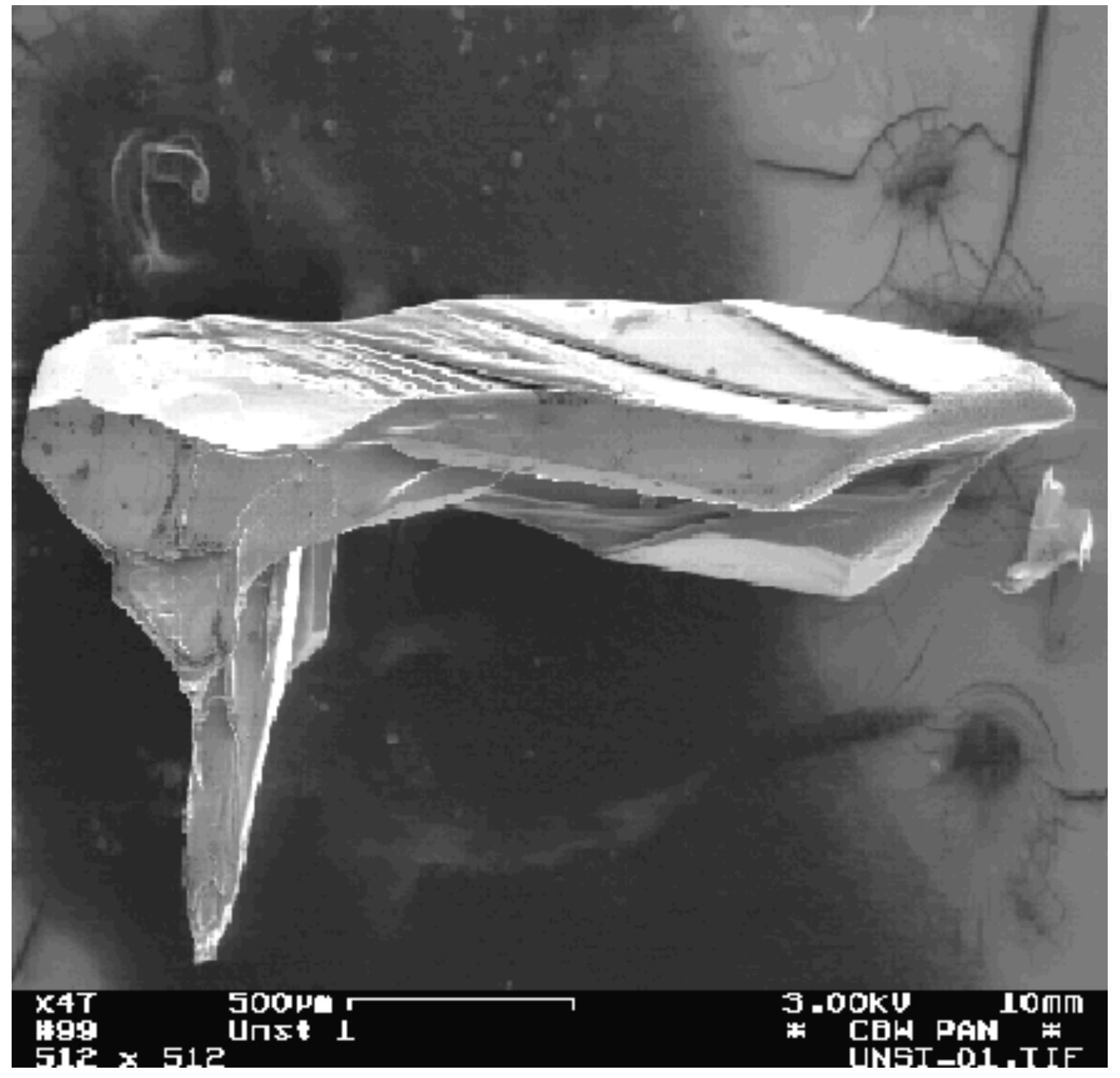

Figure $\mathbf{7 b}$. Initially plate-like growth changes into the needle-like type due to the increase of supersaturation during the growth. 


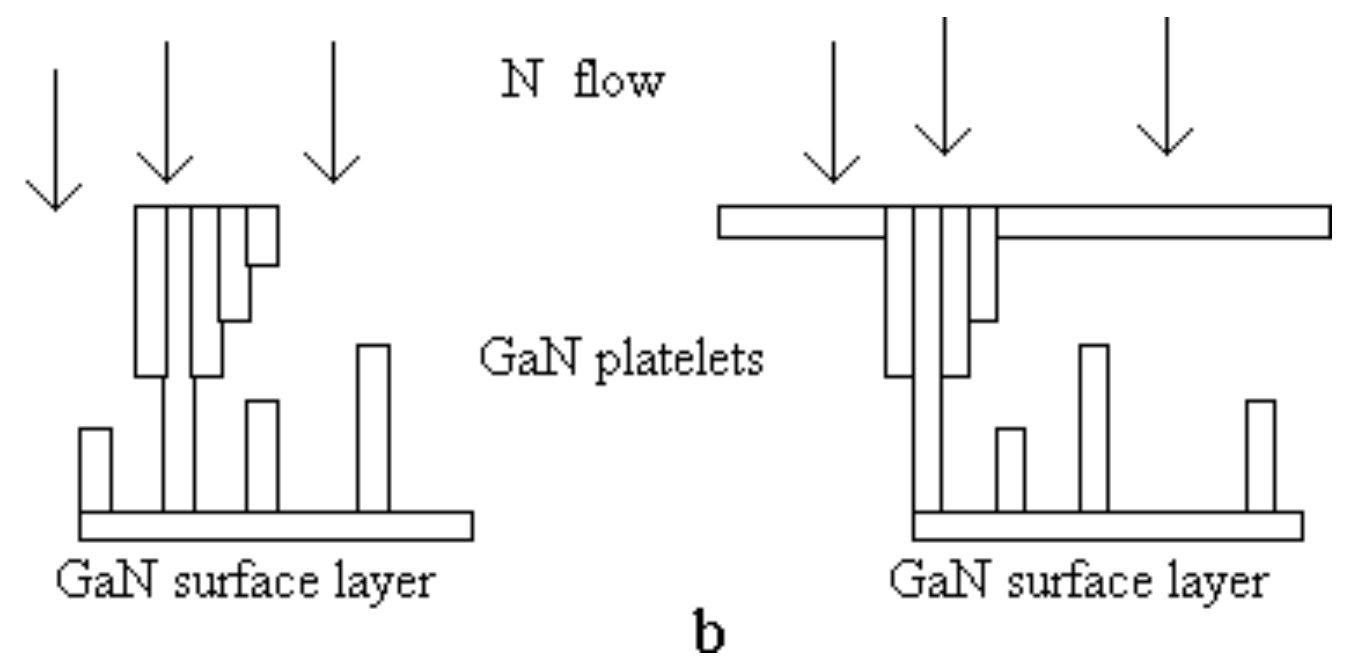

Figure 7c. Schematic of unstable growth of a GaN platelet at high, non-uniform supersaturation in the solution. (a) edge-nucleated growth on the $\{0001\}$ faces at high supersaturation, (b) initially plate-like growth changes into the needle-like type due to the increase of supersaturation during the growth.

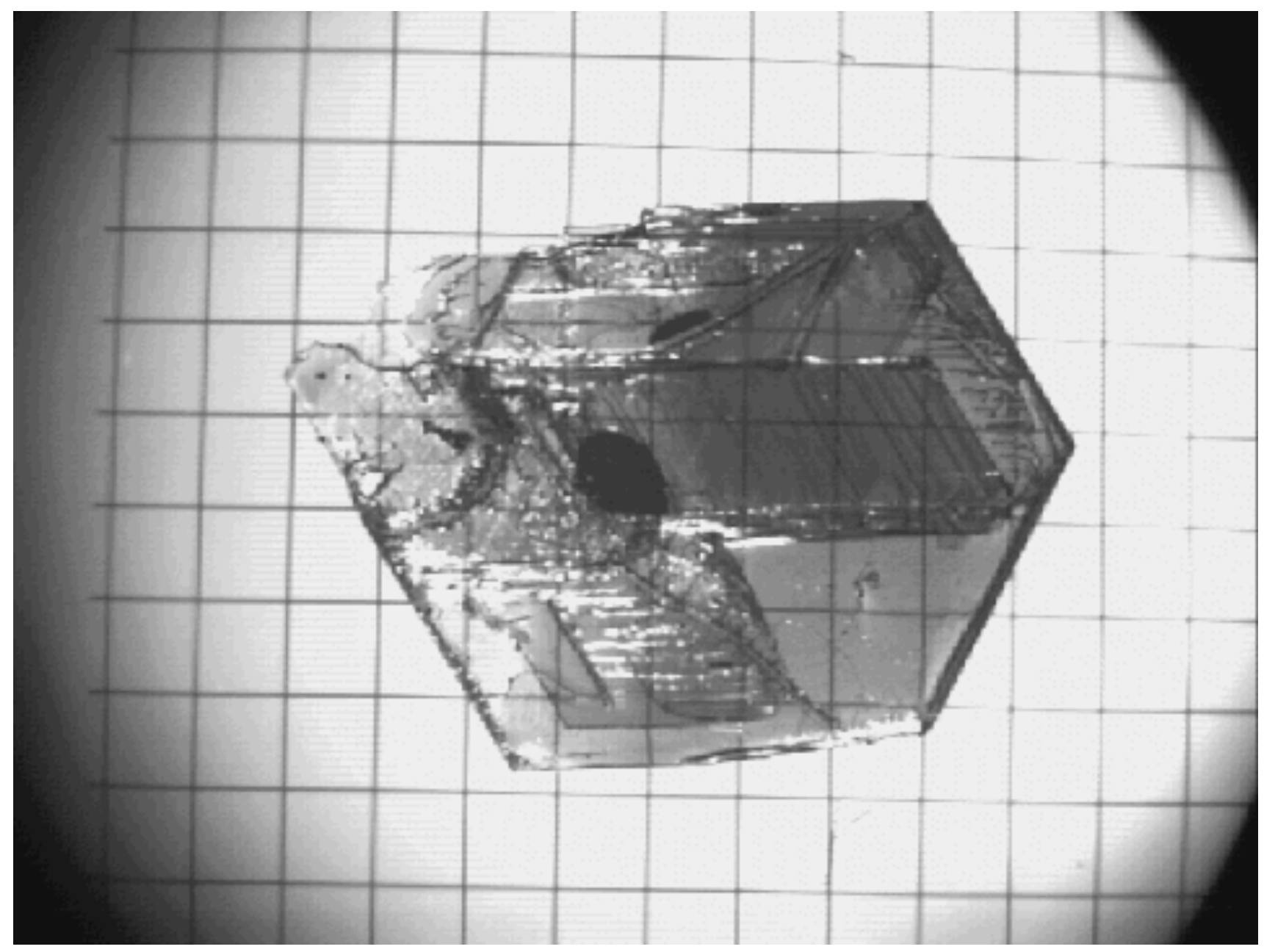

Figure 8. GaN crystal grown in a 150h process in the crucible having an ID of $17 \mathrm{~mm}$. 
fast growth direction

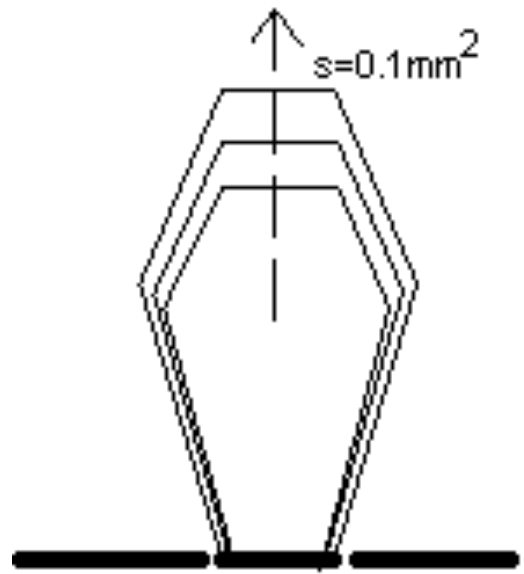

GaN surface layer
Figure 9. GaN crystal growing quickly perpendicular to the c-axis

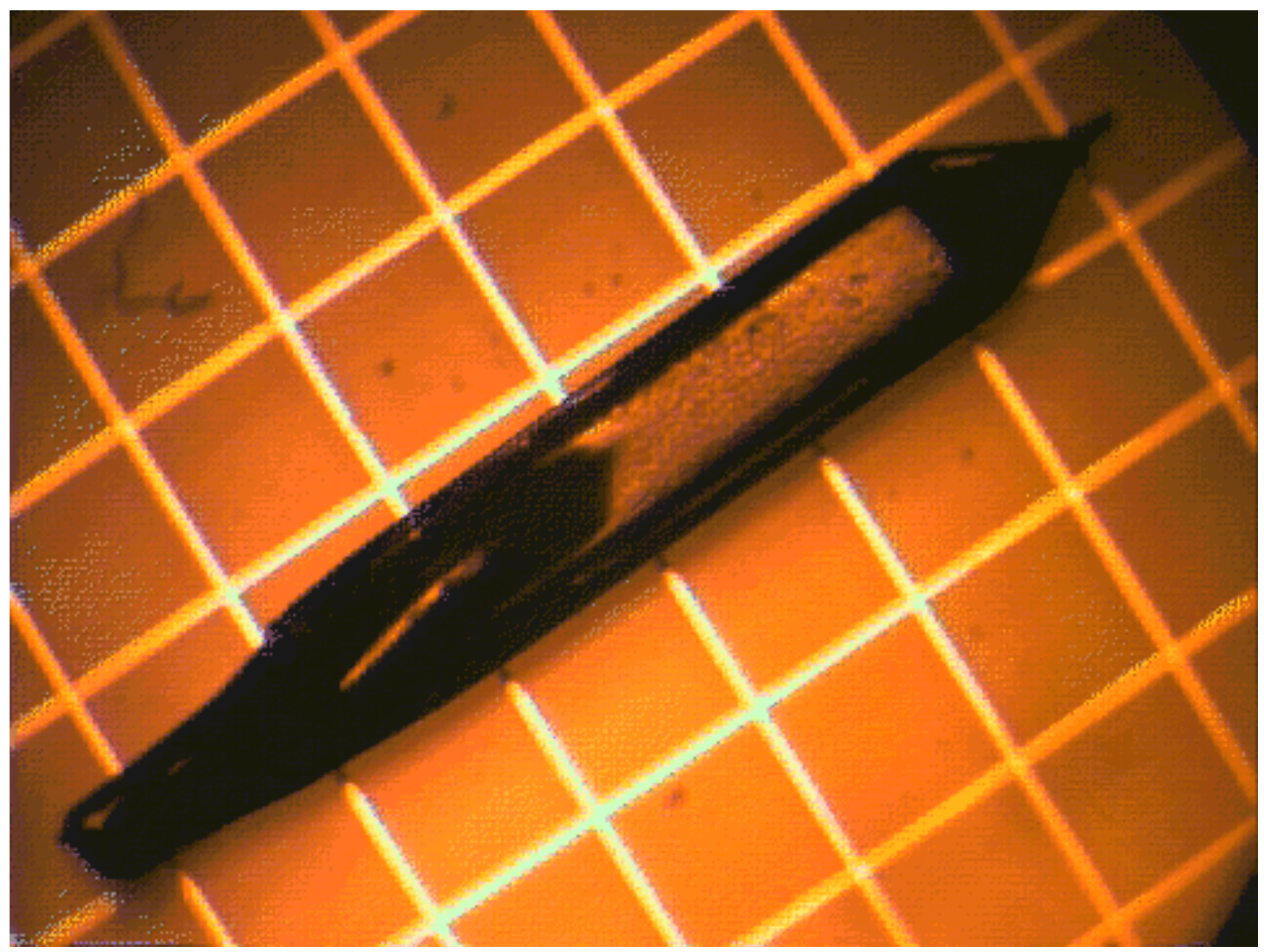

Figure 10. GaN crystal used for the intentional seeding 


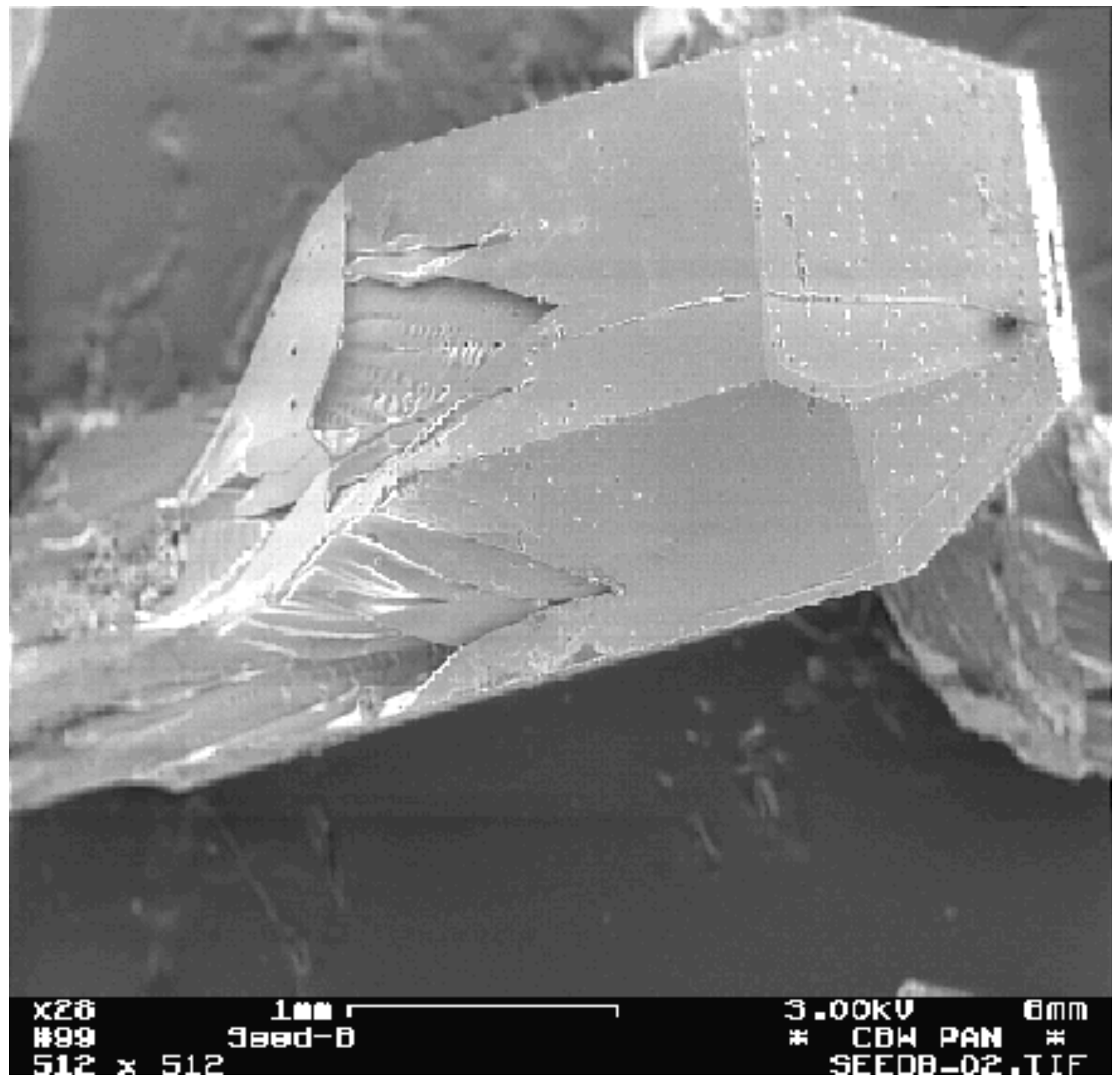

Figure 11. GaN crystal grown on a GaN single crystalline seed. 


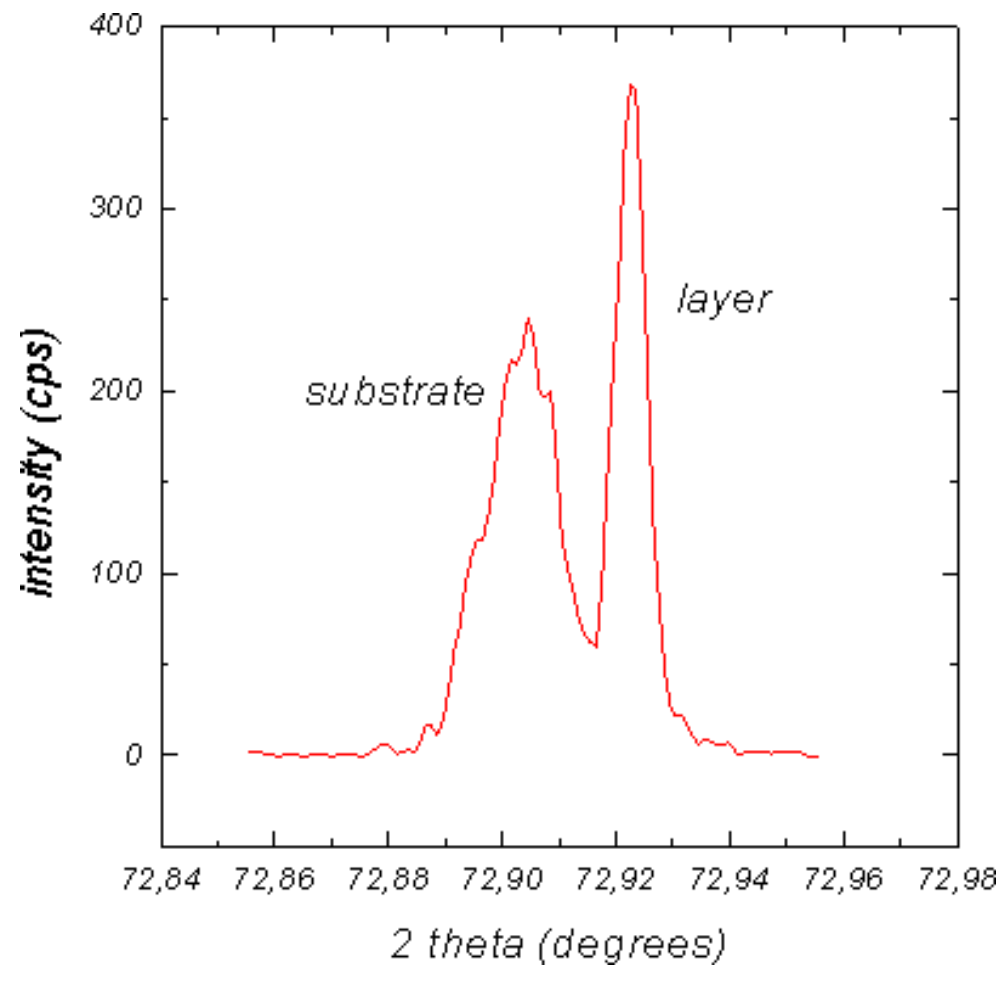

Figure 12. Diffraction peaks of (0004) CuK reflection from an MOCVD grown GaN heteroepitaxial layer and GaN substrate.

(C) 1996-1998 The Materials Research Society

\begin{tabular}{|l|l|l|l|l|}
\hline M & $\mathrm{R}$ & $\mathrm{S}$ & Internet Journal of & Nitride Semiconductor Research \\
\hline
\end{tabular}

